\title{
13. THE DIAGENETIC FACTORS CONTROLLING THE DISSOLVED ORGANIC CARBON (DOC) IN PORE WATER FROM DEEP SEA SEDIMENTS (ODP LEG 113, WEDDELL SEA) ${ }^{1}$
}

\author{
Per Kr. Egeberg ${ }^{2}$ and Mohamed I. Abdullah ${ }^{3}$
}

\begin{abstract}
Dissolved organic carbon (DOC) was determined in pore water extracted from pelagic and hemipelagic sediments recovered during Leg 113. DOC concentration varied between 1.82 and $13.6 \mathrm{mg} \mathrm{C} / \mathrm{L}$ which is one to two orders of magnitude less than previously reported for hemipelagic sediments. It is argued that this difference is related to differences in the intensity of degradation of organic matter. As a first approximation it is found that in reducing sediments, the level of DOC is proportional to the intensity of sulfate reduction. It is suggested that DOC is formed by different mechanisms in oxic and reducing environments.
\end{abstract}

\section{INTRODUCTION}

The diagenesis of organic compounds in marine sediment pore water has received little attention. The few published measurements of dissolved organic carbon (DOC) in marine sediments are mainly for coastal environments (Starikova, 1970; Nissenbaum et al., 1971; Krom and Sholkovitz, 1977; Henrichs and Farrington, 1987). Starikova (1970) and Krom and Sholkovitz (1977) observed that the concentration of DOC in oxic sediments remained relatively constant at $5-15 \mathrm{mg} \mathrm{C} / \mathrm{L}$, while in reducing sediments the concentration of DOC increased almost linearly with depth. DOC concentrations of $70 \mathrm{mg} \mathrm{C} / \mathrm{L}$ were observed within the first meter of the reducing Loch Duich sediments (a fjord-type estuary environment, Krom and Sholkovitz, 1977). In the reducing Saanich inlet sediments, Nissenbaum et al. (1971) measured concentrations of DOC between 42 and 148 $\mathrm{mg} \mathrm{C} / \mathrm{L}$.

Using ultrafiltration, Krom and Sholkovitz (1977) defined low (LMW, molecular weight less than 1000) and high molecular weight (HMW, molecular weight greater than 1000) fractions. They found that the LMW-fraction (carbohydrates, amino acids, fatty acids, etc.) constituted less than $10 \%$ of the DOC and whereas the concentration of DOC increased with depth, the concentration of LMW remained more or less constant. Using molecular absorption spectroscopy in the UV-visible range, Krom and Sholkovitz (1977) showed several similarities between the DOC and humic material. They considered the HMW fraction to have been formed by condensation of LMW compounds and concluded that in anoxic pore waters the accumulation of HMW dissolved organic matter is unaffected by the type of metabolic oxidation (sulfate reduction or methane production). On the other hand, Nissenbaum et al. (1971) demonstrated that the HMW fraction of dissolved organic matter (DOM) isolated from reducing sediments contained HMW polymers of amino acids and carbohydrates. They also showed that with increasing depth, the concentration of HMW DOM increased relative to the total DOM. Based on solid-state ${ }^{13} \mathrm{C}$ NMR studies of dissolved organic matter in pore water, Orem and Hatcher (1987) proposed the hypothesis that the HMW

\footnotetext{
${ }^{1}$ Barker, P. F., Kennett, J. P., et al., 1990. Proc. ODP, Sci. Results, 113: College Station, TX (Ocean Drilling Program).

2 University of Oslo, Department of Geology, P.O. Box 1047, Blindern, N0316 Oslo 3 , Norway.

${ }^{3}$ University of Oslo, Department of Marine Zoology and Marine Chemistry, P.O. Box 1064, Blindern, N-0316 Oslo 3, Norway.
}

DOM results from degradation of the labile fraction of the sedimentary organic matter. Thus humic and fulvic acids may act as precursors of the HMW DOM in pore water, rather than the condensation products of DOM.

To our knowledge the only studies of dissolved organic carbon in pore waters of marine sediments buried deeper than 40 $\mathrm{m}$ below seafloor (mbsf) are those reported by Michaelis et al. (1982) and Ishizuka et al. (1986b). Analyzing pore water from DSDP Sites 474 and 479 (see Table 1 for information on the individual sites), Michaelis et al. (1982) reported concentrations of dissolved organic carbon between 31 and $204 \mathrm{mg} \mathrm{C} / \mathrm{L}$. No systematic depth trends were observed. They found that free carbohydrates and amino acids constituted less than $20 \%$ of DOC and that the relative proportion of these compounds decreased with depth in the core. At ODP Sites 618, 619, and 623, Ishizuka et al (1986b) reported concentrations of DOC between 23 and $225 \mathrm{mg} \mathrm{C} / \mathrm{L}$. They observed roughly parallel concentration profiles of sugars and DOC. At Site 618 a subsurface maximum in DOC of $225 \mathrm{mg} / \mathrm{L}$ was observed at $12 \mathrm{mbsf}$, whereas at Site 619 they observed increasing DOC concentrations from 72 $\mathrm{mg} / \mathrm{L}$ at $10 \mathrm{mbsf}$ to $97 \mathrm{mg} / \mathrm{L}$ at $189 \mathrm{mbsf}$. At Site 623 the concentration remained more or less constant at about $25 \mathrm{mg} / \mathrm{L}$ from 4 to 152 mbsf.

The aim of this study is to examine the distribution of DOC in the interstitial waters of the pelagic and hemipelagic sediments recovered during ODP Leg 113, and, by correlation with other parameters and previous studies, to elucidate the mechanisms that govern the concentration of DOC.

\section{METHODS}

Core material was collected during ODP Leg 113 in the Weddell Sea between 5 January and 11 March 1987 . The interstitial waters were extracted on board using a hydraulic press and the procedure and equipment described by Manheim and Sayles (1974). The membrane filters used during squeezing were soaked in distilled deionized water for $24 \mathrm{hr}$ and then rinsed thoroughly with distilled deionized water. Subsamples were taken for determination of DOC (stored in sealed glass ampoules at $-20^{\circ} \mathrm{C}$ ) and for manganese analysis (acidified and stored in sealed teflon tubes).

Alkalinity, $\mathrm{pH}$, and ammonium were determined on board using the methods described by Gieskes (1974). Sulfate was determined by ion chromatography. The concentration of sedimentary organic carbon was determined by Rock-Eval analyses.

Back in our laboratories, dissolved manganese was determined by atomic absorption spectrophotometry. Dissolved organic carbon was determined using an automated method based on the nondispersive IRspectrophotometric quantification of $\mathrm{CO}_{2}$ evolved after wet chemical UV-/persulfate oxidation of DOM (Collins and Williams, 1977; Rykkje, 1985). Prior to oxidation the inorganic carbon was removed by 
Table 1. Location and brief description of DSDP sites used for comparison.

\begin{tabular}{|c|c|c|c|c|}
\hline & Location & $\begin{array}{l}\text { Water } \\
\text { depth } \\
(\mathrm{m})\end{array}$ & $\begin{array}{l}\text { Predominant lithologies } \\
\text { and sediment environment }\end{array}$ & References \\
\hline \multirow[t]{2}{*}{ Leg 64 Site 474} & $\begin{array}{l}\text { Baja California } \\
\text { margin }\end{array}$ & 3033 & $\begin{array}{l}\text { Predominately hemipelagic muds } \\
\text { rich in diatoms, mud turbi- } \\
\text { dites, and their more lithified } \\
\text { counterparts. Sediment envi- } \\
\text { ronment range from distal to } \\
\text { middle fan }\end{array}$ & Curray et al. (1982) \\
\hline & Guaymas Basin Slope & 747 & $\begin{array}{l}\text { Hemipelagic muddy diatom ooze } \\
\text { to mudstone. Sediment envi- } \\
\text { ronment interpreted as hemi- } \\
\text { pelagic outer slope }\end{array}$ & Curray et al. (1982) \\
\hline \multirow[t]{2}{*}{ Leg 96 Site 618} & $\begin{array}{c}\text { Gulf of Mexico } \\
\text { Orca Basin }\end{array}$ & 2412 & $\begin{array}{l}\text { Clays, muds, and silt-laminated } \\
\text { muds deposited by slumping } \\
\text { and continuous sedimentation }\end{array}$ & Bouma et al. (1986a and b) \\
\hline & $\begin{array}{l}\text { Gulf of Mexico } \\
\text { Pigmy Basin }\end{array}$ & 2259 & $\begin{array}{l}\text { Calcareous clays and muds, clays, } \\
\text { muds, silts, and sands depos- } \\
\text { ited by turbidites, and contin- } \\
\text { uous hemipelagic and pelagic } \\
\text { deposition }\end{array}$ & Bouma et al. (1986a and b) \\
\hline Site 623 & $\begin{array}{l}\text { Gulf of Mexico } \\
\text { Lower Mississippi } \\
\text { Fan }\end{array}$ & 3177 & $\begin{array}{l}\text { Clays, muds, silt-laminated muds, } \\
\text { silts, and sands, interpreted as } \\
\text { buried channels, overbank, } \\
\text { and distal turbidites }\end{array}$ & $\begin{array}{l}\text { Bouma et al. (1986a) } \\
\text { Stelting et al. (1986) }\end{array}$ \\
\hline
\end{tabular}

acidifying the samples with $0.1 \mathrm{~N} \mathrm{HCl}$ and scrubbing it with purified oxygen. Gershey et al. (1979) compared the efficiencies of the dry-combustion method of -MacKinnon (1978), the photo-oxidation/persulfate method of Collins and Williams (1977), and the persulfate oxidation procedure of Menzel and Vaccaro (1964) for determining the concentration of DOC in seawater. Of these methods, the one based on dry combustion is accepted by most analysts as complete (Wangersky, 1978; Wangersky and Zika, 1978; MacKinnon, 1978; Gershey et al, 1979; Suzuki et al., 1985). Gershey et al. (1979) found less than $5 \%$ difference between dry combustion and the UV-oxidation method whereas the persulfate method gave lower results, particularly for deep seawater samples where the dissolved organic matter is presumably more refractive. In a recent study, Sugimura et al. (1988) compared the persulfate method (not UV-/persulfate) with a catalytic high-temperature wet combustion method. They confirmed the inefficiency of the persulfate method found by Gershey et al. (1979). Although we do not have any definite proof that the method in this study gives universally $100 \%$ recovery, we do not believe that our comparatively low results are caused by poor oxidation efficiency (EDTA, urea, and other common organic compounds were completely degraded). This view is supported by the work of Armstrong et al. (1966) and Goulden and Brooksbank (1975) who observed $100 \%$ degradation of humic acid by photo-oxidation. Humic acids probably represent the most refractory compounds. High DOC values may result from contamination during drilling or from rupture of cells (bacteria, organic debris) because of the pressure drop during core retrieval. Circulating seawater is used to clean the bit during drilling. The results of the major element studies (Egeberg et al., this volume) indicate only minor invasion of seawater. Another source of contamination is from lubricants used on rotating parts. We do not have any data to assess the extent of contamination from this source. Degens and Mopper (1976) estimated that less than $1 \%$ of the organic carbon produced by local primary production is incorporated into deep-sea sediments. Thus the number of cells surviving the first meters of burial without being partly degraded is probably very small. In marine sediments the number of bacteria cells decreases rapidly with increasing depth (Price, 1976). However, we do not see any steep gradients in DOC close to the sediment surface. Hence, we do not believe that leaching of DOM from ruptured cells is a serious source of contamination in deepsea sediments.

\section{LOCATIONS AND SEDIMENT DESCRIPTION}

\section{Site 689 (Maud Rise)}

This is located near the crest of Maud Rise (Fig. 1) at a water depth of $2080 \mathrm{~m}$. The recovered sediments are pelagic, biogenic in origin, and range in age from Campanian/Maestrichtian to Pleistocene. Average rate of accumulation is $4 \mathrm{~m} / \mathrm{m}$.y.

\section{Site 690 (Maud Rise)}

This site is situated on the southwestern flank of Maud Rise in $2914 \mathrm{~m}$ of water. The sediments range in age from Late Cretaceous to Pleistocene. Rate of accumulation varies from very low (less than $2 \mathrm{~m} / \mathrm{m} . \mathrm{y}$.) to $12 \mathrm{~m} . / \mathrm{m} . \mathrm{y}$. during Pliocene. The sequence is mainly biogenic, but terrigenous and volcanic detritus becomes a significant component around $140 \mathrm{mbsf}$.

\section{Site 693 (Dronning Maud Land margin).}

This site lies on a mid-slope bench of the Weddell Sea margin of East Antarctica in $2359 \mathrm{~m}$ of water. The sediments range in age from Lower Cretaceous to Pleistocene. Due to poor core recovery and the presence of several hiatuses, estimates of rates of accumulation are uncertain. Between 14 and 129 mbsf, the rates vary from 14 to $46 \mathrm{~m} / \mathrm{m}$.y. The sediments consist of $400 \mathrm{~m}$ of hemipelagic mud overlaying claystone, mudstone, diatomite, and silicified sandstone.

\section{Site 694 (Weddell abyssal plain)}

Site 694 is situated on the northern part of the Weddell abyssal plain in $4653 \mathrm{~m}$ of water. The recovered sediments range in age from middle Miocene to Pleistocene and are mostly terrigenous with a minor biosiliceous component. Rates of accumulation are highly variable with an average of $3 \mathrm{~m} / \mathrm{m}$.y. in the upper $14 \mathrm{mbsf}$ increasing to at least $180 \mathrm{~m} / \mathrm{m}$.y. between 21 and $112 \mathrm{mbsf}$.

\section{Site 695 (South Orkney microcontinent)}

Site 695 was located on the southeastern edge of the South Orkney microcontinent in $1305 \mathrm{~m}$ of water. The sediments are biosiliceous hemipelagic deposits and range in age from Miocene/Pliocene to Pleistocene. Estimated rates of accumulation increase from less than $5 \mathrm{~m} / \mathrm{m}$.y. in Pleistocene to $215 \mathrm{~m} / \mathrm{m}$.y. in early Pliocene, below which rates of $105 \mathrm{~m} / \mathrm{m} . \mathrm{y}$. are found.

\section{Site 696 (South Orkney microcontinent)}

This is situated on the southeastern margin of the South Orkney microcontinent in $650 \mathrm{~m}$ of water. The sediments are 


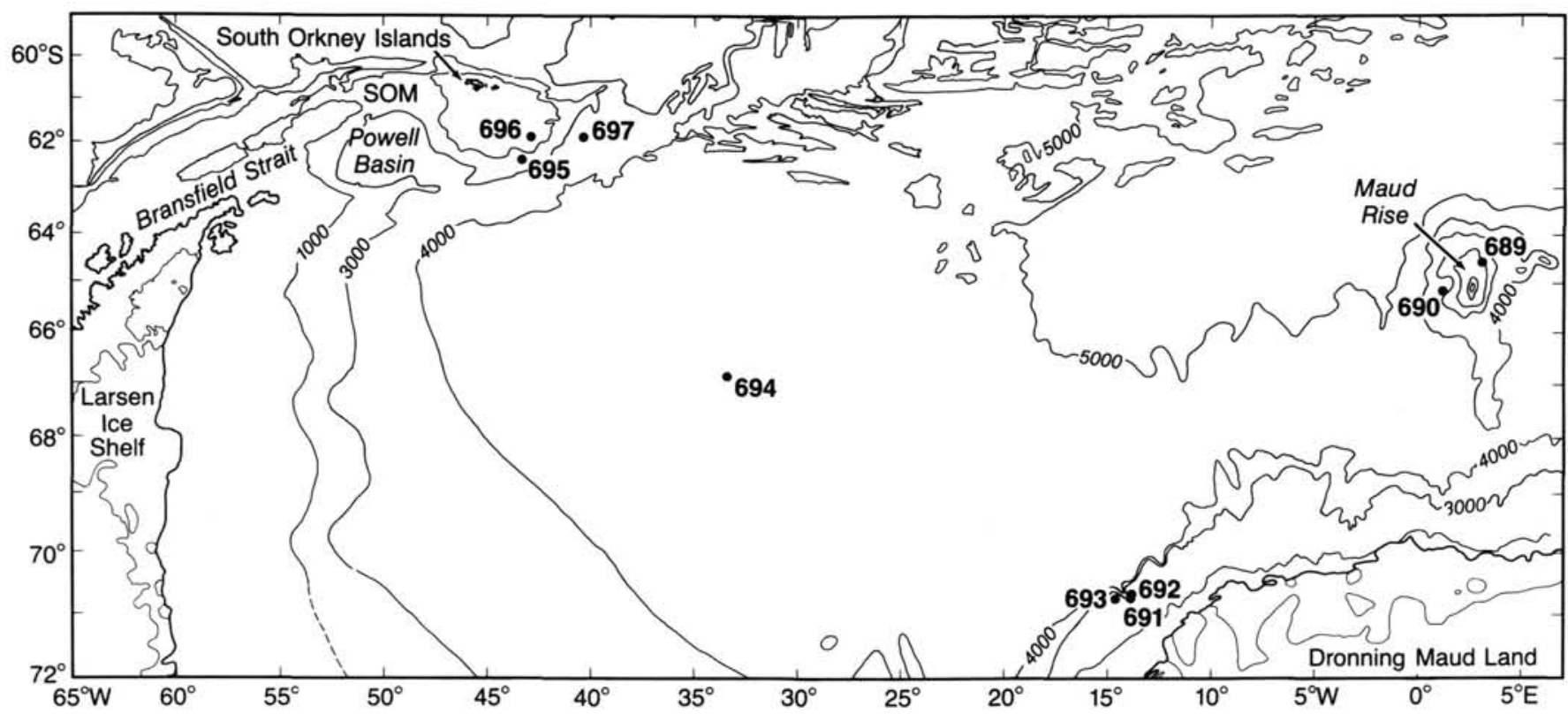

Figure 1. ODP Leg 113 Site locations. SOM = South Orkney microcontinent.

terrigenous, hemipelagic, and pelagic and range in age from middle/upper Eocene to Pleistocene. The rate of accumulation increases from an average of $5 \mathrm{~m} / \mathrm{m} . \mathrm{y}$. in Pleistocene to $18 \mathrm{~m} /$ m.y. in middle Pliocene and $120 \mathrm{~m} / \mathrm{m}$.y. in early Pliocene, while the Eocene sequence is characterized by an overall accumulation rate of $6 \mathrm{~m} / \mathrm{m}$.y. Due to very poor recovery, rates in the Miocene and Oligocene are difficult to determine.

\section{Site 697 (South Orkney microcontinent-Jane Basin)}

This site is located in Jane Basin under $3484 \mathrm{~m}$ of water. The recovered sediments are hemipelagic with a minor biosiliceous component, and range in age from Pliocene to Pleistocene. Rates of accumulation increase from $40 \mathrm{~m} / \mathrm{m}$.y. in the upper $100 \mathrm{mbsf}$ (Pleistocene and upper Pliocene) to $125-150 \mathrm{~m} / \mathrm{m}$.y. at depths between 250 and 300 mbsf (lower Pliocene).

\section{RESULTS}

The analytical data for pore water are listed in Tables 2-8. The concentration of DOC ranges from 1.8 to $13.6 \mathrm{mg} \mathrm{C} / \mathrm{L}$. The average concentration at each site are listed in Table 9. Site 697 is the only site at which the distribution of DOC varies systematically with depth (Fig. 2). The profile (Site 697) shows a pronounced subsurface maximum of $13.6 \mathrm{mg} \mathrm{C} / \mathrm{L}$ at $90.1 \mathrm{mbsf}$ superimposed on an overall decrease from $7.63 \mathrm{mg} \mathrm{C} / \mathrm{L}$ at 19.45 mbsf to $2.55 \mathrm{mg} \mathrm{C} / \mathrm{L}$ at $305.5 \mathrm{mbsf}$. There is a general tendency for higher observed concentrations of DOC in pore waters sampled from the shallower sections (Tables 2-8).

The average concentration of sedimentary organic carbon at the hemipelagic sites $(693,694,695,696,697)$ is $0.39 \%$. The claystone found between 427 and 465 mbsf at Site 693 contains an average of $2.56 \%$ organic carbon. At the pelagic Site 689 , the average concentration of organic carbon in the upper $49 \mathrm{~m}$ is $0.11 \%$ and is below detection limit $(0.01 \%)$ at greater depths. No site exhibited systematic variations of sedimentary organic carbon with depth. The average concentrations of sedimentary organic carbon at each site are listed in Table 9.

The concentration of sulfate varied between seawater values and zero (Fig. 3). The most extensive sulfate reduction was observed in the hemipelagic sediments recovered from sites on the northern margin of the Weddell Sea $(695,696,697)$. At the pelagic sites (689 and 690) only minor sulfate reduction was observed while the sites on the Antarctic margin (693) and in the center of the Weddell Sea (694) were intermediate.

The alkalinity and concentration of ammonium varied from 2.1 to $18.4 \mathrm{meq} / \mathrm{L}$ and from 0.01 to $1.8 \mathrm{mmol}$ respectively. Alkalinity and ammonia correlated inversely with sulfate.

Table 2. Concentration of dissolved manganese, alkalinity, dissolved organic carbon, sulfate, ammonium, pH, and concentration of sedimentary organic carbon at Site 689.

\begin{tabular}{crrrrrrr}
\hline $\begin{array}{l}\text { Core, section, } \\
\text { interval (cm) }\end{array}$ & $\begin{array}{c}\text { Depth } \\
(\mathrm{mbsf})\end{array}$ & $\begin{array}{c}\mathrm{Mn}^{2+} \\
(\mathrm{ppm})\end{array}$ & $\mathrm{pH}$ & $\begin{array}{c}\mathrm{Alk} \\
(\mathrm{meq} / \mathrm{L})\end{array}$ & $\begin{array}{c}\mathrm{DOC} \\
(\mathrm{mgC} / \mathrm{L})\end{array}$ & $\begin{array}{c}\mathrm{C}_{\text {org }} \\
(\mathrm{wt} \%)\end{array}$ & $\begin{array}{c}\mathrm{SO}_{4} \\
(\mathrm{mM})\end{array}$ \\
\hline $113-689 \mathrm{~B}-1 \mathrm{H}-2,135-142$ & 2.85 & & & & & & 29.5 \\
$1 \mathrm{H}-3,78-85$ & 3.78 & & & & 5.16 & & 29.2 \\
$1 \mathrm{H}-3,145-150$ & 4.45 & 0.08 & 7.82 & 2.91 & & 0.11 & 29.5 \\
$2 \mathrm{H}-2,66-70$ & 7.46 & & & & & & 28.9 \\
$3 \mathrm{H}-4,120-125$ & 20.50 & 0.08 & 7.65 & 3.19 & 4.29 & 0.13 & 28.0 \\
$6 \mathrm{H}-4,120-125$ & 49.00 & 0.07 & 7.66 & 3.15 & & 0.09 & 28.3 \\
$9 \mathrm{H}-4,120-125$ & 77.80 & 0.36 & 7.31 & 2.90 & & 0 & 26.7 \\
$12 \mathrm{H}-4,115-125$ & 106.65 & 0.32 & 7.66 & 3.10 & & 0 & 26.7 \\
$15 \mathrm{H}-4,115-125$ & 135.55 & 0.12 & 7.78 & 3.06 & 1.82 & 0 & 28.0 \\
$18 \mathrm{H}-4,115-125$ & 164.45 & 0.05 & 7.81 & 3.10 & & 0 & 25.7 \\
$22 \mathrm{X}-4,115-125$ & 203.15 & 0.04 & 7.48 & 2.43 & 6.76 & 0 & 26.0 \\
$25 \mathrm{X}-4,115-125$ & 232.25 & 0.05 & 7.54 & 2.35 & & 0 & 26.8 \\
$28 \mathrm{X}-3,115-125$ & 259.72 & 0.05 & 7.57 & 2.06 & 2.84 & 0 & 26.3 \\
\hline
\end{tabular}


Table 3. Concentration of dissolved manganese, alkalinity, dissolved organic carbon, sulfate, ammonium, pH, and concentration of sedimentary organic carbon at Site 690 .

\begin{tabular}{|c|c|c|c|c|c|c|c|c|}
\hline $\begin{array}{l}\text { Core, section, } \\
\text { interval }(\mathrm{cm})\end{array}$ & $\begin{array}{l}\text { Depth } \\
\text { (mbsf) }\end{array}$ & $\begin{array}{l}\mathrm{Mn}^{2+} \\
(\mathrm{ppm})\end{array}$ & $\mathrm{pH}$ & $\begin{array}{c}\text { Alk } \\
(\mathrm{meq} / \mathrm{L})\end{array}$ & $\begin{array}{c}\text { DOC } \\
(\mathrm{mgC} / \mathrm{L})\end{array}$ & $\begin{array}{c}\mathrm{C}_{\mathrm{org}} \\
(\mathrm{wt} \%)\end{array}$ & $\begin{array}{c}\mathrm{SO}_{4} \\
(\mathrm{mM})\end{array}$ & $\begin{array}{r}\mathrm{NH}_{4} \\
(\mathrm{mM})\end{array}$ \\
\hline $113-690 \mathrm{~B}-2 \mathrm{H}-3,145-150$ & 6.55 & 0.08 & 7.70 & 2.84 & 5.01 & 0.01 & 29.2 & 0.10 \\
\hline $3 \mathrm{H}-4,120-125$ & 17.40 & 0.05 & 7.52 & 2.93 & 2.10 & 0.07 & 29.2 & 0.94 \\
\hline $6 \mathrm{H}-4,120-125$ & 46.50 & 0.02 & 7.96 & 3.11 & 2.55 & 0.18 & 27.6 & 0.01 \\
\hline $9 \mathrm{H}-4,120-125$ & 75.50 & 0.04 & 7.81 & 3.75 & & 0.07 & 26.5 & 0.06 \\
\hline $12 \mathrm{H}-5,120-125$ & 106.00 & 0.04 & 7.69 & 3.79 & 2.98 & 0.04 & 24.7 & 0.05 \\
\hline $16 \mathrm{H}-4,120-125$ & 143.50 & 0.08 & 7.90 & 4.04 & & 0.07 & 22.9 & 0.01 \\
\hline $19 \mathrm{H}-4,115-125$ & 172.55 & 0.08 & 7.83 & 3.82 & 6.91 & 0.26 & 22.3 & 0.08 \\
\hline $22 \mathrm{H}-3,120-125$ & 189.40 & 0.10 & 7.77 & 3.84 & & 0.08 & 24.6 & 0.12 \\
\hline $25 \mathrm{H}-4,115-125$ & 209.85 & 0.10 & 7.74 & 3.87 & 2.11 & 0.02 & 22.7 & 0.01 \\
\hline $113-690 C-11 X-3,115-125$ & 208.35 & 0.10 & 7.95 & 3.27 & & 0.19 & 21.9 & 0.04 \\
\hline $14 X-4,115-125$ & 238.85 & 0.12 & 7.75 & 3.87 & & 0.03 & 20.1 & 1.04 \\
\hline $17 X-2,115-125$ & 264.45 & 0.13 & 7.63 & 3.47 & 2.55 & 0.36 & 22.8 & 0.33 \\
\hline $20 \times-4,115-125$ & 296.45 & 0.16 & 7.86 & 3.10 & 2.98 & 0.53 & 21.0 & 0.66 \\
\hline
\end{tabular}

Table 4. Concentration of dissolved manganese, alkalinity, dissolved organic carbon, sulfate, ammonium, pH, and concentration of sedimentary organic carbon at Site 693.

\begin{tabular}{|c|c|c|c|c|c|c|c|c|}
\hline $\begin{array}{l}\text { Core, section, } \\
\text { interval (cm) }\end{array}$ & $\begin{array}{l}\text { Depth } \\
\text { (mbsf) }\end{array}$ & $\begin{array}{l}\mathrm{Mn}^{2+} \\
(\mathrm{ppm})\end{array}$ & $\mathrm{pH}$ & $\underset{(\mathrm{meq} / \mathrm{L})}{\text { Alk }}$ & $\underset{(\mathrm{mgC} / \mathrm{L})}{\mathrm{DOC}}$ & $\begin{array}{c}C_{\mathrm{org}} \\
(\mathrm{wt} \%)\end{array}$ & $\begin{array}{l}\mathrm{SO}_{4} \\
(\mathrm{mM})\end{array}$ & $\begin{array}{l}\mathrm{NH}_{4} \\
(\mathrm{mM})\end{array}$ \\
\hline $113-693 \mathrm{~A}-2 \mathrm{R}-4,145-150$ & 8.45 & 0.07 & 7.90 & 3.90 & 9.81 & 0.10 & 28.6 & 0.10 \\
\hline $4 \mathrm{R}-3,120-125$ & 26.10 & 3.03 & 7.83 & 3.56 & & 0.25 & 27.7 & 0.17 \\
\hline $6 \mathrm{R}-4,120-125$ & 46.80 & 2.02 & 7.76 & 3.49 & 6.47 & 0.10 & 27.8 & 0.26 \\
\hline $9 \mathrm{R}-4,120-125$ & 75.80 & 2.34 & 8.10 & 3.75 & & 0.16 & 25.1 & 0.09 \\
\hline $12 \mathrm{R}-4,120-125$ & 104.60 & 2.63 & 8.15 & 3.88 & 2.84 & 0.20 & 25.6 & 0.27 \\
\hline $18 \mathrm{R}-3,120-125$ & 161.10 & 2.39 & 8.19 & 3.36 & & 0 & 22.7 & 0.20 \\
\hline $25 \mathrm{R}-2,120-125$ & 227.30 & 1.86 & 8.07 & 3.32 & 4.00 & 0 & 20.3 & 0.29 \\
\hline $113-693 \mathrm{~B}-2 \mathrm{X}-3,145-150$ & 238.25 & 1.34 & 8.03 & 2.79 & & & 21.3 & 0.28 \\
\hline $113-693 \mathrm{~A}-28 \mathrm{R}-3,120-125$ & 257.80 & 1.34 & 8.10 & 3.14 & 7.64 & & 19.3 & 0.22 \\
\hline $113-693 \mathrm{~B}-6 \mathrm{X}-4,145-150$ & 273.75 & 1.45 & 7.98 & 2.99 & & & 18.6 & 0.36 \\
\hline $9 X-3,120-125$ & 301.00 & 1.28 & 8.24 & 3.19 & 4.00 & & 19.1 & 0.31 \\
\hline $12 \mathrm{X}-3,120-125$ & 330.00 & 1.09 & 7.76 & 2.27 & 6.62 & & 17.8 & 0.24 \\
\hline $15 X-2,140-150$ & 357.60 & 1.27 & 8.34 & 3.45 & 2.25 & & 18.1 & 0.33 \\
\hline $113-693 \mathrm{~A}-40 \mathrm{R}-2,138-142$ & 371.78 & 1.46 & 8.16 & 3.02 & & & 17.2 & 0.87 \\
\hline $113-693 \mathrm{~B}-17 \mathrm{X}-1,145-150$ & 375.45 & 1.11 & 8.28 & 2.83 & 3.42 & & 17.2 & 0.35 \\
\hline $19 \mathrm{X}-2,140-150$ & 396.30 & 1.20 & 8.20 & 3.55 & & & 16.9 & 0.32 \\
\hline $113-693 \mathrm{~A}-48 \mathrm{R}-2,120-125$ & 448.10 & 1.14 & 8.16 & 2.83 & & 2.30 & 16.0 & 0.62 \\
\hline
\end{tabular}

Table 5. Concentration of dissolved manganese, alkalinity, dissolved organic carbon, sulfate, ammonium, pH, and concentration of sedimentary organic carbon at Site 694.

\begin{tabular}{ccccccccc}
\hline $\begin{array}{c}\text { Core, section, } \\
\text { interval }(\mathrm{cm})\end{array}$ & $\begin{array}{c}\text { Depth } \\
(\mathrm{m})\end{array}$ & $\begin{array}{c}\mathrm{Mn}^{2+} \\
(\mathrm{ppm})\end{array}$ & $\mathrm{pH}$ & $\begin{array}{c}\mathrm{Alk} \\
(\mathrm{meq} / \mathrm{L})\end{array}$ & $\begin{array}{c}\mathrm{DOC} \\
(\mathrm{mgC} / \mathrm{L})\end{array}$ & $\begin{array}{c}\mathrm{C}_{\mathrm{org}} \\
(\mathrm{wt} \%)\end{array}$ & $\begin{array}{c}\mathrm{SO}_{4} \\
(\mathrm{mM})\end{array}$ & $\begin{array}{c}\mathrm{NH}_{4} \\
(\mathrm{mM})\end{array}$ \\
\hline $113-694 \mathrm{~B}-1 \mathrm{H}-2,145-150$ & 2.95 & 0.09 & 7.91 & 3.10 & 6.47 & 0.25 & 28.1 & 0.01 \\
$3 \mathrm{H}-2,120-125$ & 17.79 & 0.13 & 7.79 & 2.75 & 2.84 & 0.26 & 28.3 & 0.01 \\
$22 \mathrm{X}-1,145-150$ & 151.85 & 4.78 & 7.91 & 3.07 & 3.71 & 0.75 & 23.0 & 0.27 \\
$24 \mathrm{X}-2,120-125$ & 172.30 & 5.46 & 7.99 & 3.40 & & 0.10 & 21.7 & 0.20 \\
& & & & & & & & \\
$113-694 \mathrm{C}-5 \mathrm{X}-1,145-150$ & 210.35 & 3.29 & 8.38 & 3.15 & 6.33 & 0.14 & 20.6 & 0.40 \\
$14 \mathrm{X}-4,115-125$ & 300.35 & 1.59 & 8.44 & 3.00 & & 0.60 & 18.3 & 0.55 \\
$19 \mathrm{X}-2,145-150$ & 345.95 & 1.18 & 8.20 & 3.55 & 10.6 & 0.50 & 16.2 & 0.61 \\
$22 \mathrm{X}-2,115-125$ & 374.65 & 0.99 & 8.20 & 3.01 & 3.42 & & 15.4 & 1.42 \\
\hline
\end{tabular}

The concentration of dissolved manganese varied between $0.02 \mathrm{ppm}$ at $46.5 \mathrm{mbsf}$ (Site 690) and $16.8 \mathrm{ppm}$ at $5.95 \mathrm{mbsf}$ (Site 697). Pronounced subsurface maxima were observed at each site with the exception of Site 690 . The concentration of dissolved manganese is an order of magnitude lower at the pelagic sites (689 and 690) than that found at the hemipelagic sites.

\section{DISCUSSION}

Previous studies of coastal (Starikova, 1970; Nissenbaum et al., 1971; Krom and Sholkovitz, 1977) and hemipelagic sediments (Michaelis et al., 1982; Ishizuka et al., 1986b) have demonstrated that the bulk of dissolved organic carbon in marine pore waters is made up of complex HMW compounds. Nissen- 
Table 6. Concentration of dissolved manganese, alkalinity, dissolved organic carbon, sulfate, ammonium, pH, and concentration of sedimentary organic carbon at Site 695 .

\begin{tabular}{ccccccccc}
\hline $\begin{array}{c}\text { Core, section, } \\
\text { interval }(\mathrm{cm})\end{array}$ & $\begin{array}{c}\text { Depth } \\
(\mathrm{m})\end{array}$ & $\begin{array}{c}\mathrm{Mn}^{2+} \\
(\mathrm{ppm})\end{array}$ & $\mathrm{pH}$ & $\begin{array}{c}\mathrm{Alk} \\
(\mathrm{meq} / \mathrm{L})\end{array}$ & $\begin{array}{c}\mathrm{DOC} \\
(\mathrm{mgC} / \mathrm{L})\end{array}$ & $\begin{array}{c}\mathrm{C}_{\mathrm{org}} \\
(\mathrm{wt} \%)\end{array}$ & $\begin{array}{c}\mathrm{SO}_{4} \\
(\mathrm{mM})\end{array}$ & $\begin{array}{c}\mathrm{NH}_{4} \\
(\mathrm{mM})\end{array}$ \\
\hline $113-695 \mathrm{~A}-1 \mathrm{H}-3,145-150$ & 4.45 & 0.06 & 7.86 & 3.72 & 2.40 & & 29.7 & 0.00 \\
$3 \mathrm{H}-4,140-145$ & 18.10 & 0.08 & 7.92 & 3.78 & & 0.43 & 29.4 & 0.11 \\
6H-3, 120-125 & 45.40 & 0.40 & 8.00 & 5.23 & 4.87 & 0.16 & 28.3 & 0.34 \\
$9 \mathrm{H}-2,120-125$ & 72.70 & 0.72 & 8.00 & 7.07 & & 0.44 & 24.1 & 0.61 \\
$12 \mathrm{H}-4,120-125$ & 94.90 & 0.76 & 8.11 & 7.95 & & & 21.9 & 0.86 \\
$15 \mathrm{H}-3,120-125$ & 122.40 & 0.63 & 8.40 & 8.14 & & 0.40 & 19.0 & 0.97 \\
$19 \mathrm{X}-4,120-125$ & 153.00 & 0.80 & 7.78 & 9.35 & 4.58 & 0.56 & 17.6 & 1.17 \\
$22 \mathrm{X}-3,120-125$ & 180.70 & 0.95 & 7.78 & 9.62 & & 0.35 & 14.1 & 1.31 \\
$25 \mathrm{X}-3,120-125$ & 200.10 & 0.94 & 7.84 & 9.86 & & 0.48 & 13.8 & 1.33 \\
$29 \mathrm{X}-2,115-125$ & 237.35 & 1.55 & 8.17 & 10.99 & & 0.37 & 9.3 & 1.64 \\
$32 \mathrm{X}-4,115-125$ & 259.75 & 1.68 & 8.30 & 11.10 & 2.25 & 0.32 & 7.7 & 1.76 \\
$36 \mathrm{X}-2,115-125$ & 295.45 & 0.55 & 7.91 & 12.32 & & 0.39 & 5.3 & 1.61 \\
$39 \mathrm{X}-3,115-125$ & 316.15 & 0.47 & 7.75 & 12.17 & 3.56 & 0.39 & 1.8 & 1.64 \\
$41 \mathrm{X}-3,140-150$ & 335.80 & 0.94 & 8.08 & 12.37 & & 0.24 & 1.2 & 1.57 \\
\hline
\end{tabular}

Table 7. Concentration of dissolved manganese, alkalinity, dissolved organic carbon, sulfate, ammonium, pH, and concentration of sedimentary organic carbon at Site 696.

\begin{tabular}{|c|c|c|c|c|c|c|c|c|}
\hline $\begin{array}{l}\text { Core, section, } \\
\text { interval }(\mathrm{cm})\end{array}$ & $\begin{array}{l}\text { Depth } \\
(\mathrm{m})\end{array}$ & $\begin{array}{l}\mathrm{Mn}^{2+} \\
(\mathrm{ppm})\end{array}$ & $\mathrm{pH}$ & $\begin{array}{c}\text { Alk } \\
(\mathrm{meq} / \mathrm{L})\end{array}$ & $\begin{array}{c}\text { DOC } \\
(\mathrm{mgC} / \mathrm{L})\end{array}$ & $\begin{array}{c}\mathrm{C}_{\mathrm{org}} \\
\text { (wt\%) }\end{array}$ & $\begin{array}{c}\mathrm{SO}_{4} \\
(\mathrm{mM})\end{array}$ & $\begin{array}{l}\mathrm{NH}_{4} \\
(\mathrm{mM})\end{array}$ \\
\hline $113-696 \mathrm{~A}-2 \mathrm{H}-3,120-125$ & 6.70 & 0.08 & 7.90 & 3.94 & & 0.29 & 26.6 & 0.12 \\
\hline $5 \mathrm{H}-3,120-125$ & 35.40 & 1.23 & 7.94 & 3.84 & & 0.48 & 17.3 & 0.46 \\
\hline $8 \mathrm{H}-4,120-125$ & 65.70 & 1.39 & 8.21 & 4.40 & 2.69 & 0.46 & 12.0 & 0.80 \\
\hline 113-696B-3R-3, 120-125 & 90.40 & 1.42 & 8.23 & 3.73 & & 0.45 & 15.3 & 0.86 \\
\hline $6 \mathrm{R}-3,120-125$ & 119.40 & 1.41 & 8.14 & 4.14 & 3.13 & 0.25 & 6.4 & 1.13 \\
\hline $20 \mathrm{R}-2,120-125$ & 233.80 & 0.66 & 7.84 & 2.64 & & 0.23 & 5.3 & 1.46 \\
\hline $26 \mathrm{R}-2,120-125$ & 291.70 & 0.32 & 7.84 & 2.64 & 4.44 & 0.42 & 7.9 & 1.40 \\
\hline $34 \mathrm{R}-1,120-125$ & 367.50 & 0.45 & 7.67 & 3.07 & & & 5.5 & 1.69 \\
\hline $53 \mathrm{R}-4,115-125$ & 554.55 & 0.58 & 8.34 & 2.65 & 11.2 & 0.94 & 0.1 & 1.74 \\
\hline
\end{tabular}

Table 8. Concentration of dissolved manganese, alkalinity, dissolved organic carbon, sulfate, ammonium, pH, and concentration of sedimentary organic carbon at Site 697.

\begin{tabular}{|c|c|c|c|c|c|c|c|c|}
\hline $\begin{array}{l}\text { Core, section, } \\
\text { interval }(\mathrm{cm})\end{array}$ & $\begin{array}{l}\text { Depth } \\
\text { (m) }\end{array}$ & $\begin{array}{l}\mathrm{Mn}^{2+} \\
(\mathrm{ppm})\end{array}$ & $\mathrm{pH}$ & $\begin{array}{c}\text { Alk } \\
(\mathrm{meq} / \mathrm{L})\end{array}$ & $\begin{array}{c}\text { DOC } \\
(\mathrm{mgC} / \mathrm{L})\end{array}$ & $\begin{array}{c}\mathrm{C}_{\mathrm{org}} \\
(\mathrm{wt} \%)\end{array}$ & $\begin{array}{l}\mathrm{SO}_{4} \\
(\mathrm{mM})\end{array}$ & $\begin{array}{l}\mathrm{NH}_{4} \\
(\mathrm{mM})\end{array}$ \\
\hline $113-697 \mathrm{~A}-1 \mathrm{H}-4,145-150$ & 5.95 & 16.80 & 7.85 & 4.89 & & 0.34 & 28.0 & 0.10 \\
\hline 113-697B-1H-1, $145-150$ & 19.45 & 3.21 & 7.93 & 9.48 & 7.63 & 0.38 & 23.1 & 0.44 \\
\hline $3 \mathrm{H}-4,120-125$ & 42.80 & 3.38 & 7.80 & 12.00 & & 0.43 & 19.9 & 0.56 \\
\hline $4 \mathrm{H}-4,115-125$ & 52.45 & 3.93 & 7.82 & 14.56 & 6.62 & 0.37 & 16.8 & 0.71 \\
\hline $5 \mathrm{H}-3,115-125$ & 60.65 & 3.76 & & & & & & \\
\hline $6 \mathrm{H}-2,115-125$ & 68.85 & 4.53 & 7.94 & 16.64 & 8.65 & 0.41 & 13.1 & 0.87 \\
\hline $7 \mathrm{H}-2,140-150$ & 78.90 & 4.05 & 7.89 & 16.11 & & 0.40 & 13.1 & 0.82 \\
\hline $8 \mathrm{H}-3,140-150$ & 90.10 & 3.91 & 8.10 & 17.53 & 13.1 & & 10.3 & 1.07 \\
\hline $9 \mathrm{H}-3,115-125$ & 99.55 & 3.81 & 8.06 & 17.35 & & 0.33 & 10.3 & 1.03 \\
\hline $13 \mathrm{X}-3,115-125$ & 132.75 & 3.41 & 7.78 & 18.35 & 6.33 & 0.42 & 6.5 & 1.10 \\
\hline $16 \times-4,115-125$ & 163.15 & 3.04 & 7.74 & 17.74 & & 0.32 & 6.2 & 1.00 \\
\hline $19 \times-3,115-125$ & 190.75 & 2.16 & 7.84 & 14.00 & 5.60 & 0.43 & 5.3 & 0.92 \\
\hline $22 X-2,115-125$ & 218.15 & 2.09 & 7.99 & 13.77 & & 0.29 & 5.0 & 1.11 \\
\hline $26 \mathrm{X}-2,115-125$ & 256.85 & 2.42 & 7.89 & 11.08 & 3.85 & 0.30 & 5.9 & 0.87 \\
\hline $29 \times-2,115-125$ & 285.90 & 2.19 & 7.93 & 11.57 & & 0.37 & 4.7 & 0.83 \\
\hline $31 X-2,140-150$ & 305.50 & 2.52 & 8.27 & 10.07 & 2.55 & & 7.0 & 1.07 \\
\hline
\end{tabular}

baum et al. (1971) and Krom and Sholkovitz (1977) argued that the HMW compounds are formed by condensation of LMW compounds released during biological degradation of sedimentary organic carbon. In a subsequent study, Nissenbaum and Kaplan (1972) showed that "fulvic" and "humic" compounds extracted from marine sediments are compositionally and structurally different from their terrestrial counterparts. They suggested that continuous polymerization of DOM leads to the formation of insoluble fulvic acids. Thus the amount of DOC in any body of water may be considered to reflect the balance between rate of generation and rate of polymerization.
Both Starikova (1970) and Krom and Sholkovitz (1977) found that the most important factor governing the level of pore water DOC is the redox conditions of the subsurface environment. Several of the LMW compounds that may condense to HMW DOC are also potential energy sources for heterothrophs (e.g., sugars, fatty acids, amino acids). Thus the low levels of DOC in oxic sediments may be a result of much faster turnover. An alternative mechanism may be deduced from the experiments conducted by Harvey et al. (1983). They showed that the condensation of polyunsaturated lipids to a humic compound involves oxidative cross-linking. Thus the low levels of DOC in oxic pore 
Table 9. Average concentration of DOC, sedimentary organic carbon, and maximum depth and age for Leg 64, Leg 96, and Leg 113 sites.

\begin{tabular}{|c|c|c|c|}
\hline Location & $\begin{array}{l}\text { Maximum } \\
\text { age/depth } \\
\text { (m.y./mbsf) }\end{array}$ & $\begin{array}{c}\text { DOC } \\
(\mathrm{mgC} / \mathrm{L})\end{array}$ & $\begin{array}{c}\mathrm{C}_{\mathrm{org}} \\
(w t \%)\end{array}$ \\
\hline \multicolumn{4}{|c|}{ Leg 113 (Weddell Sea) } \\
\hline Site 689 & $70 / 260$ & 4.2 & ${ }^{\mathrm{a}} 0.11$ \\
\hline Site 690 & $72 / 296$ & 3.4 & 0.16 \\
\hline Site 693 & $40 / 448$ & 5.2 & $\mathrm{~b}_{0.17}$ \\
\hline Site 694 & $20 / 376$ & 5.6 & 0.39 \\
\hline Site 695 & $5 / 316$ & 3.5 & 0.37 \\
\hline Site 696 & $20 / 554$ & 5.5 & 0.49 \\
\hline Site 697 & $4 / 306$ & 6.0 & 0.37 \\
\hline
\end{tabular}

Leg 64 (Gulf of California)

$\begin{array}{cccc}\text { Site } 474 & c_{3 / 546} & d_{73.1} & e, f, g_{1.36} \\ \text { Site } 479 & c_{2 / 367} & 66.7 & e, 8_{2.8}\end{array}$

Leg 96 (Gulf of Mexico)

$\begin{array}{llrr}\text { Site 618 } & { }^{\mathrm{h}} 0.08 / 91 & { }^{\mathrm{i}}{ }_{108.1} & \mathrm{j}_{0.79} \\ \text { Site 619 } & \mathrm{h}_{0.18 / 189} & { }^{\mathrm{i}_{77.8}} & \mathrm{j}_{0.66} \\ \text { Site 623 } & 0.06 / 152 & { }^{\mathrm{i}} 26.3 & { }^{\mathrm{k}} 1.02\end{array}$

\footnotetext{
In the upper $49 \mathrm{~m}$.

b In the upper $370 \mathrm{~m}$.

c Curray et al. (1982).

d Michaelis et al. (1982).

e Galimov et al. (1982).

f Rullkotter et al. (1982).

$\mathrm{g}$ Deroo et al. (1982).

${ }^{h}$ Bouma et al. (1986b).

i Ishizuka et al. (1986a).

${ }^{j}$ Whelan et al. (1986).

${ }^{k}$ Whelan and Tarafa (1986).
}

waters may also be due to more rapid polymerization favored by the higher concentration of oxidizing agents.

Manganese is the most redox-sensitive compound measured in this study. Data in Tables 2-8 show that at all sites except Site
690 subsurface maxima of dissolved manganese were observed, indicating that the redox conditions at depth are sufficiently reducing to stabilize manganese as Mn(II). Taking the reducing conditions into account, it is reasonable to assume that despite the low concentration of sedimentary organic matter the sulfate profiles (Fig. 3) reflect an ongoing sulfate reduction. With the exception of Site 695, each of the profiles may be interpreted as parts of exponential curves. At present we have no explanation for the nearly linear profile at Site 695 . However, the abrupt increase in concentration of methane at the depths where sulfate is exhausted (Sites 695 and 696; Barker et al., 1988) suggests that the conditions remain viable for further microbial activity.

In Table 9 we compare our data with the DOC data given by Michaelis et al. (1982) and Ishizuka et al. (1986b). The "maximum depths" refer to deepest sample on which analyses DOC in pore water were made, and "maximum ages" refer to the ages of these samples.

Inspection of Table 9 reveals that the levels of DOC determined in Leg 113 sediments are one to two orders of magnitude less than those found in Legs 64 and 96 sediments.

The low level of DOC found in this study is in marked contrast to what was found in the pore water of Leg 64 (Michaelis et al., 1982) and Leg 96 (Ishizuka et al., 1986b) (Table 9). In the light of the discussion given above, this difference is probably related to the fact that Leg 113 sediments contain significantly lower concentrations of sedimentary organic material and that they are deposited at considerably lower rates (Table 9). The high rates of sedimentation and the high concentrations of sedimentary organic carbon give rise to more extensive sulfate reduction at Sites 474 and 479 (Gieskes et al., 1982) and at Sites 618 and 619 (Ishizuka et al., 1986a) as compared to the Leg 113 sites. Site 623 is an exception. Here, despite a rate of accumulation of $2500 \mathrm{~m} / \mathrm{m} . \mathrm{y}$. and a content of organic carbon of about $1 \%$ (Table 9), the sulfate profile shows no evidence of sulfate reduction. However, the elevated alkalinity levels (Ishizuka et al., 1986a) are indicative of microbial activity. Whelan (1986) attributed the anomalous sulfate profile at Site 623 to unknown processes.

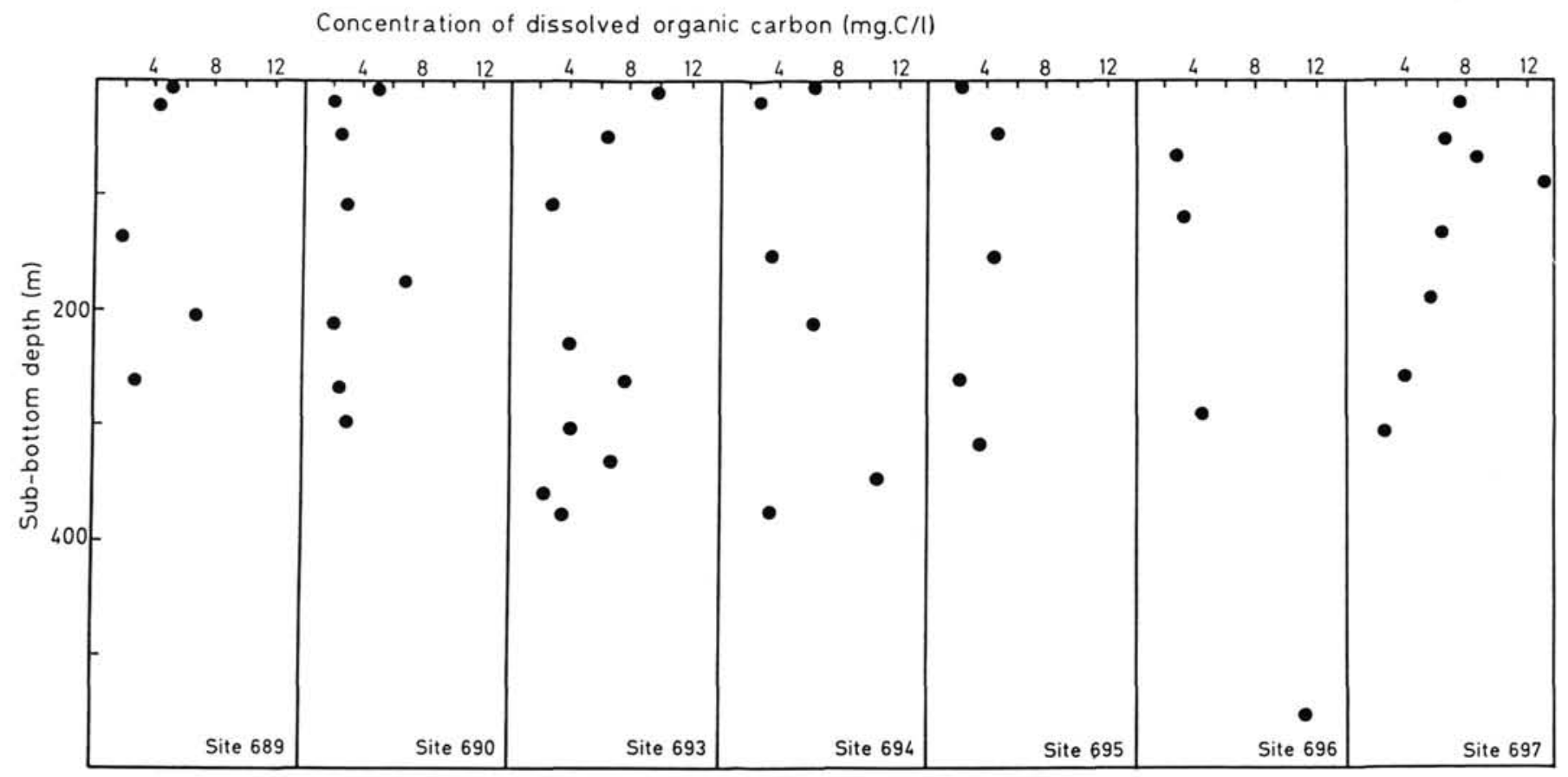

Figure 2. Pore water concentrations of dissolved organic carbon. Data from Tables 2-8. 


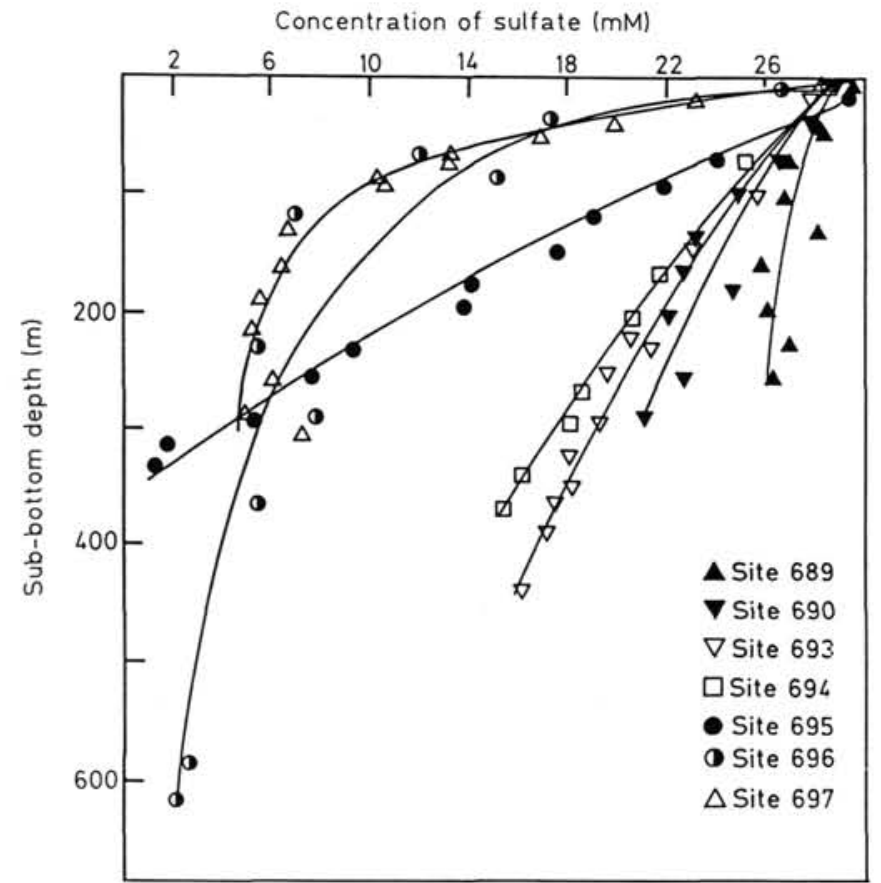

Figure 3. Pore water concentrations of sulfate. Data from Tables 2-8.

In an effort to delineate the controlling mechanism we hypothesize that the amount of HMW DOC produced is proportional to the rate of breakdown of sedimentary organic matter. This may be stated by the following equation:

$$
\mathrm{DOC}=\mathrm{K}_{1} \cdot \mathrm{R}
$$

where DOC is the concentration of dissolved carbon, $K_{1}$ is a constant, and $\mathrm{R}$ is the rate (intensity) of sulfate reduction. The reasoning being that for LMW organic compounds to condense to form HMW DOC, they must be generated at a sufficiently high rate to escape further bacterial oxidation. Furthermore, we assume that the main agent of degradation of sedimentary organic matter is sulfate-reducing bacteria. Also, equation (1) is only valid when the rate of polymerization to insoluble compounds is very much lower than the rate of generation of LMW. Based on the information available to him, Berner (1980, p. 88) assumed that the geopolymerization to insoluble compounds is very rapid and is essentially completed near the sediment/water interface. However, because most of the DOC is produced near the sediment/water interface where microbial activity is most intense, the observations of high concentrations of DOC in deeply buried pore water (e.g., $204 \mathrm{mg} / \mathrm{L}$ at $546 \mathrm{mbsf}$ at Site 474 and $41 \mathrm{mg} / \mathrm{L}$ at $367 \mathrm{mbsf}$ at Site 479 , Michaelis et al., 1982) seem to support our assumption.

Toth and Lerman (1977) and Berner (1978) noted that, where $\mathrm{K}$ is the rate constant for sulfate reduction (assuming first-order kinetics) and $w$ is the rate of deposition, there is a linear correlation between $(\mathrm{K} / \mathrm{w})$ and $\mathrm{w}$, that is

$$
\mathrm{K} / \mathrm{W}=\mathrm{K}_{3} \cdot \mathrm{W}
$$

where $K_{3}$ is an empirical constant. Berner (1978) argued that qualitatively the above relationship implies that high rates of deposition results in more rapid burial and better preservation of readily metabolizable compounds which, at slower rates of deposition, would otherwise be consumed by the benthic fauna. This relationship also incorporates the effect of diffusion of sul- fate across the sediment/water interface. Based on these assumption, we attempt the following derivation. For first-order kinetics the rate of sulfate reduction $(R)$ is defined as:

$$
\mathrm{R}=\mathrm{K} \cdot \mathrm{G}
$$

where $\mathrm{G}$ is the concentration of metabolizable sedimentary organic matter, and $\mathrm{K}$ is the rate constant. By rearranging equation (2) and substituting equation (2) and (3) into equation (1), we arrive at the following relationship:

$$
\mathrm{DOC} / \mathrm{G}=\mathrm{K}_{2} \cdot \mathrm{w}^{2}
$$

where $K_{2}$ is the product of $K_{1}$ and $K_{3}$.

Thus, if our hypothesis is correct, and the concentration of metabolizable organic carbon (G) is proportional to the observed concentration of sedimentary organic carbon $(C)$, we would expect a linear relationship between the $\log (\mathrm{DOC} / \mathrm{C})$ and $\log (w)$ with a slope of 2 . For this analysis, we restrict ourselves to those sites where the sulfate observations show evidence of sulfate reduction close to the sediment/water interface (Sites $474,479,618,619,695,696,697)$.

The data from Table 9 are presented as a $\log / \log$ plot in Figure 4 . For constructing this figure we have used an average rate of accumulation obtained by dividing the depth of the deepest sample by its age. At first sight this may appear to be an unreasonable approach. However, because diffusion will smooth out variations caused by varying rates of accumulation, this may be justified for this crude hypothesis. Figure 4 shows that the data from Leg 113 (Sites 695, 696, 696) and Leg 64 (Sites 474, 479)

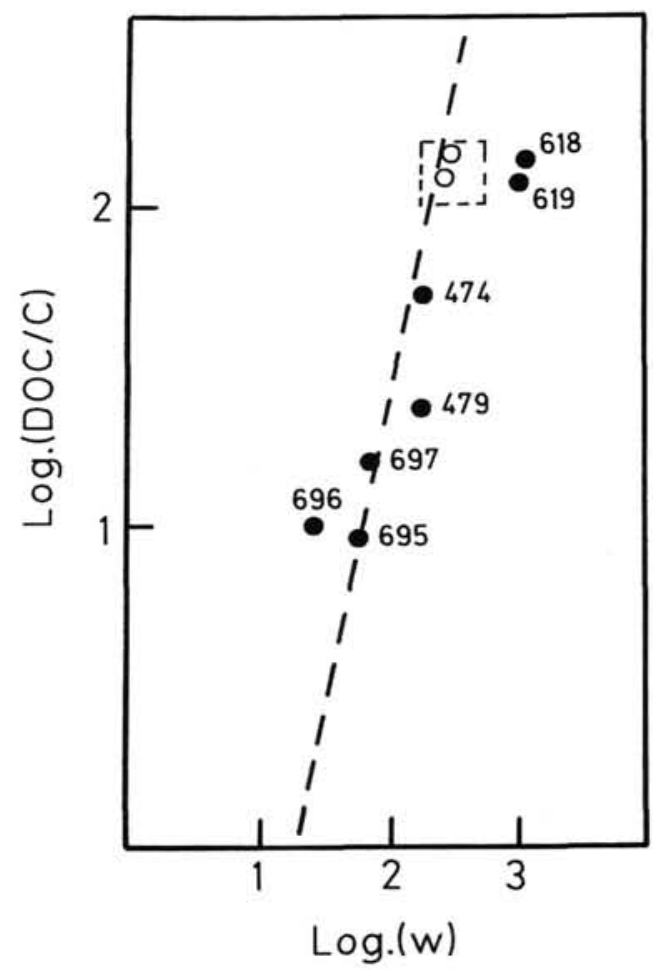

Figure 4. Logarithm of the ratio of the concentration of dissolved organic matter and the concentration of sedimentary organic carbon vs. the logarithm of the rate of accumulation. Solid symbols, data from Leg 64, Leg 96, and Leg 113 (Table 9); open symbols, data from Leg 96 tentatively corrected for the effect of turbidites on the rate of accumulation. 
define a trend roughly parallel to that predicted by our hypothesis. The data from Leg 96 (Sites 618, 619) are displaced toward high rates of accumulation. This offset may be due to a higher proportion of metabolizable organic carbon at these sites. However, in order to displace the data by one-half logarithmic unit, the amount of metabolizable organic carbon relative to the concentration of sedimentary organic carbon would have to be more than three times higher at the Leg 96 sites as compared to the other sites. There are no data to support this assumption. A more likely explanation may be deduced from Figure 5 showing the relationship between the average concentration of sedimentary organic carbon and the average rate of accumulation. The two lines in Figure 5 were taken from Stein (1986). It is interesting to note that all of the data from Leg 113 and Leg 64 plot in the field Stein (1986) defined on the basis of data from Holocene and Quaternary sediments. This gives credit to our implicit assumption that the observed concentration of sedimentary organic carbon is not very different from the concentration at the time of deposition. (A discussion of what implications this observation has for interpretation of organic carbon profiles is beyond the scope of this presentation.) Another interesting feature of Figure 5 of immediate relevance to this study is the fact that the Leg 96 sites $(618,619,623)$ plot outside the area, being displaced toward high rates of accumulation. When Stein (1986) established the two lines shown in Figure 5, he excluded areas with turbidites. The offset of the Leg 96 sites is probably related to the high intensity of turbidites in this region of the Gulf of Mexico (Bouma et al., 1986b). Turbidites were also observed at Site 474 (Curray et al., 1982) and at Site 694 (Barker et al., 1988). For some reason, the effect on the organic carbon/accumulation rate relationship seem to be less pronounced. Because the relationship expressed by equation (4) is based on a uniform rate of accumulation, ideally we should correct our average rates of accumulation for the effect of instantaneous deposition of turbidites, the presence of which gives an apparent high rate of accumulation and hence the shift in the plot. However, it is very difficult to quantify the amount of turbidites in poorly recovered sections, and is probably not justified considering the

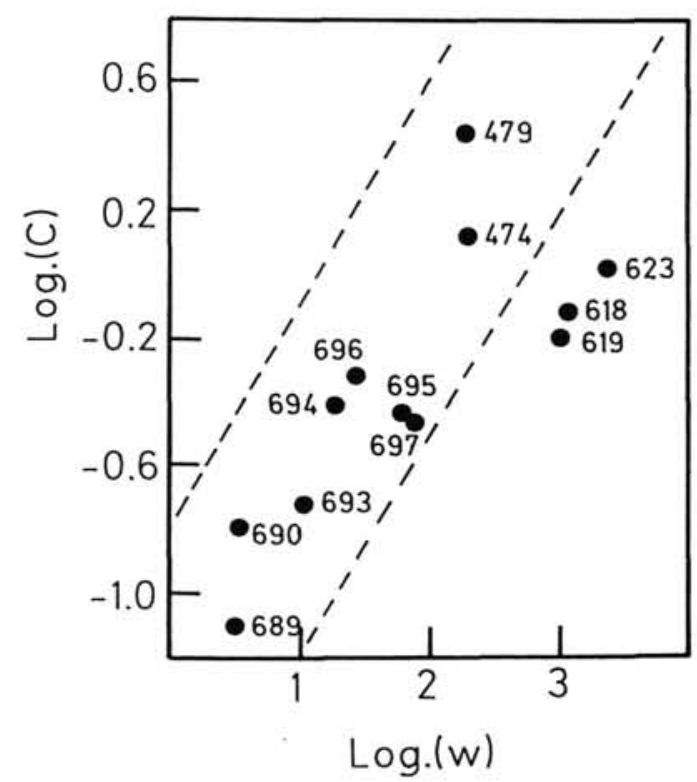

Figure 5. Correlation between concentration of sedimentary organic carbon and average rate of accumulation. The field defined by the two lines represents data from Quaternary cores and surface sediments deposited in oxic bottom water (Stein, 1986). Data from Table 9. crudeness of our hypothesis. Here it suffices to say that if the rates could be adjusted, the data from Leg 96 (Sites 618, 619) might well plot along the same line as defined by the data from Leg 113 (Sites 695, 696, 697) and Leg 64 (Sites 474, 479) (Fig. 4).

The reasonably good correspondence between this simple hypothesis (stated by equation 1) and the observations suggests that in reducing sediments, as a first approximation, the level of DOC may be considered as being a function of the intensity of sulfate reduction. This indicates that in these environments, the rate of polymerization to insoluble compounds is slow.

Inspection of Figure 2 reveals that the concentration of DOC at the less reducing sites (Sites $689,690,693,694$ ) is not very different from the concentration of DOC at the sites included in the above analysis (Sites 695, 696, 697). It is generally held that anaerobic conditions are more favorable to the preservation of organic material than aerobic conditions. This explanation is often used to account for the differences between the organic carbon content of oxic and anoxic sediments. However, this assumption has been questioned by Stumm and Morgan (1981) who favor the concept that organically enriched environments become by necessity anoxic. Whatever is the explanation, it is reasonable to assume that because of the activity of the macroand meiofauna, in oxic sediments a smaller proportion of the metabolizable organic material is degraded by extracellular enzymes. Thus, smaller amounts of LMW DOC are released to the pore water. We believe that the DOC at the sites where sulfate reduction only takes place at depth (Sites 689, 690, 693, 694) may have been formed predominantly by the pathway suggested by Orem and Hatcher (1987), that is, by solubilization of fulvic- and humic-like compounds. This idea is supported by the fact that DOM from oxic and reducing pore water has a different composition. DOM from reducing environments contains a higher proportion of carbohydrates (Orem and Hatcher, 1987). In light of the above discussion, it is not surprising that the data from Sites $689,690,693$, and 694 could not be fitted by our model (hypothesis).

\section{CONCLUSIONS}

DOC in pore water recovered from pelagic and hemipelagic sediments was found to be lower than previously reported from hemipelagic sediments. To explain the DOC variation in pore water, a simple hypothesis is postulated in terms of the various diagenetic conditions.

Based on the hypothesis that DOC is primarily HMW compounds generated through condensation reactions involving LMW products of microbiological activities, the concentration of DOC may be expressed as a function of the rate of sulfate reduction. When parameters which determine this rate, such as sedimentary organic carbon and accumulation rate, are included, it is possible to test this hypothesis using simple sedimentary observations. This procedure shows good agreement between the predicted trend and observations within a particular sedimentary domain (e.g., reducing), thus, as a first approximation, verifying the initial assumptions. An interesting corollary is that the rate of polymerization of DOM to insoluble geopolymers is a slow process at the temperatures encountered in this study (less than $40^{\circ} \mathrm{C}$ ). It is hypothesized that DOC is produced by different mechanisms depending on the redox conditions of the sediment. More data are needed to apply and test this hypothesis over a wider range of conditions, particularly under conditions of less-extensive sulfate reduction.

\section{ACKNOWLEDGMENT}

Financial support from the Norwegian Research Council for Sciences and the Humanities is gratefully acknowledged. 


\section{REFERENCES}

Armstrong, F.A.J., Williams, P. M., and Strickland, J.D.H., 1966. Photo-oxidation of organic matter in seawater by ultra-violet radiation, analytical and other applications. Nature, 211:481-483.

Barker, P. F., Kennett, J. P., et al., 1988. Proc. ODP, Init. Repts., 113: College Station, TX (Ocean Drilling Program).

Berner, R. A., 1978. Sulfate reduction and the rate of deposition of marine sediments. Earth Planet. Sci. Lett., 37:492-498.

Berner, R. A., 1980. Early Diagenesis, a Theoretical Approach: New Jersey (Princeton Univ. Press).

Bouma, A. H., Coleman J. M, Meyer, A. W. et al., 1986a. Init. Repts. $D S D P$, 96: Washington (U.S. Govt. Printing Office).

Bouma, A. H., Stelting, C. E., Coleman, J. M., Cremer, M., Droz, L. I., Meyer, A. W., Normark, W. R., O'Connell, S., Pickering, K. T., and Stow, D.A.V., 1986b. Seismic stratigraphy and sedimentary processes in Orca and Pigmy basins. In Bouma, A.H., Coleman, J.M., Meyer, A. W., et al., Init. Repts. DSDP, 96: Washington (U.S. Govt. Printing Office), 563-576.

Collins, K. J., and Williams, P. J. Le B., 1977. An automated photochemical method for the determination of dissolved organic carbon in sea and estuarine waters. Mar. Chem., 5:123-141.

Curray, J. R., Moore, D. G., et al., 1982. Init. Repts. DSDP, 64: Washington (U.S. Govt. Printing Office).

Degens, E. T., and Mopper, K., 1976. Factors controlling the distribution and early diagesis of organic material in marine sediments. In Riley J. P., and Chester, R. (Eds.) Chemical Oceanography (Vol. 6): London (Academic Press): 59-113.

Deroo, G., Herbin, J. F., Roucache, J., Boudou, J. P., Robert, P., Jardine, S., and Marestang, P., 1982. Geochemical and optical study of organic matter in some Pleistocene and Pliocene sediment from the Gulf of California: Leg 64, Holes 474 to 481A. In Curray, J. R., Moore, D. G., et al., Init. Repts. DSDP, 64: Washington (U.S. Govt. Printing Office), 855-864.

Galimov, E. M., Kodina, L. A., Bogacherva, M. P., and Shirinsky, V. G., 1982. Organic geochemical studies of samples from Deep Sea Drilling Project Leg 64, Gulf of California, Sites 474, 477, 478, 479, and 481. In Curray, J. R., Moore, D. G., et al., Init. Repts. DSDP, 64: Washington (U.S. Govt. Printing Office), 819-836.

Gershey, R. M., MacKinnon, M. D., Williams, P. J. Le B., and Moore, R. M., 1979. Comparison of three oxidation methods used for the analysis of the dissolved organic carbon in seawater. Mar. Chem., 7: 289-306.

Gieskes, J. M., 1974. Interstitial water studies, Leg 25. In Simpson, E.S.W., Schlich, R., et al., Init. Repts. DSDP, 25: Washington (U.S. Govt. Printing Office), 361-394.

Gieskes, J. M., Elderfield, H., Lawrence, R., Johnson, J., Meyers, B., and Campbell, A., 1982. Geochemistry of interstitial waters and sediments, Leg 64, Gulf of California. In Curray, J. R., Moore, D. G., Init. Repts. DSDP, 64: Washington (U.S. Govt. Printing Office), 675-694.

Goulden, P. D., and Brooksbank, P., 1975. Automated determination of dissolved organic carbon in lake water. Anal. Chem., 47:19431946.

Harvey, G. R., Boran, D. B., Chesal, L. A., and Tokar, J. M., 1983. The structure of marine fulvic and humic acids. Mar. Chem., 2:119-132.

Henrichs, S. M., and Farrington, J. W., 1987. Early diagenesis of amino acids and organic matter in two coastal marine sediments. Geochim. Cosmochim. Acta, 51:1-15.

Ishizuka, T., Kawahata, H., and Aoki, S., 1986a. Interstitial water geochemistry and clay mineralogy of the Mississippi Fan and Orca and Pigmy Basins, Deep Sea Drilling Project Leg 96. In Bouma, A. H., Coleman, J. M., Meyer, A. W., et al., Init. Repts. DSDP, 96: Washington (U.S. Govt. Printing Office), 711-728.

Ishizuka, T., Ittekkot, V., Degens, E. T., and Kawahata, H., 1986b. Preliminary data on dissolved organic carbon and sugar in interstitial water from the Mississippi Fan and Orcha and Pigmy Basins, Deep Sea Drilling Project Leg 96. In Bouma, A. H., Coleman, J. M., Meyer, A. W., et al., Init. Repts. DSDP, 96: Washington (U.S. Govt. Printing Office), 729-732.

Krom, M. D., and Sholkovitz, E. R., 1977. Nature and reaction of dissolved organic matter in the interstitial waters of marine sediments. Geochim. Cosmochim. Acta, 41:1565-1573.
MacKinnon. M. D., 1978. A dry oxidation method for the analysis of the TOC in seawater. Mar. Chem., 7:17-37.

Manheim, F. T., and Sayles, F. L., 1974. Composition and origin of interstitial waters of marine sediments based on deep sea drilling cores. In Goldberg, E. D. (Ed.), The Sea (Vol. 5): New York (Wiley Interscience), 527-568.

Menzel, D. W., and Vaccaro, R. F., 1964. The measurement of dissolved organic and particulate carbon in seawater. Limnol. Oceanogr. 9: 138-142.

Michaelis, W., Mycke, B., Vogt, J., Schuetze, G., and Degens, E. T., 1982. Organic geochemistry of interstitial waters, Sites 474 and 479 , Leg 64. In Curray, J. R., Moore, D. G., et al., Init. Repts. DSDP, 64: Washington (U.S. Govt. Printing Office), 933-938.

Nissenbaum, A., Baedecker, M. J., and Kaplan, I. R., 1971. Studies on dissolved organic matter from interstitial water of a reducing fjord. In Gaertner, H. R. and Wehner, H. (Eds.), Advances in Organic Geochemistry (1971): Oxford (Pergamon Press) 427-440.

Nissenbaum, A., and Kaplan, I. R., 1972. Chemical and isotopic evidence for the in situ origin of marine humic substances. Limnol. Oceanogr., 17:570-582.

Orem, W. H., and Hatcher, P. G., 1987. Solid-state ${ }^{13} \mathrm{C}$ NMR studies of dissolved organic matter in pore waters from different depositional environments. Geochim. Cosmochim. Acta, 11:73-82.

Price, N. B., 1976 Chemical diagenesis in sediments. In Riley, J. P., and Chester, R. (Eds.), Chemical Oceanography (Vol. 6), London (Academic Press): 1-58.

Rullkotter, J., von der Dick, H., and Welte, D. H., 1982. Organic petrography and extractable hydrocarbons of sediments from the Gulf of California, Deep Sea Drilling Project, Leg 64. In Curray, J. R., Moore, D. G., et al., Init. Repts. DSDP, 64: Washington (U.S. Govt. Printing Office), 837-854.

Rykkje, J. M., 1985. Dissolved organic matter in sea water. (Cand. Real. thesis) University of Oslo.

Starikova, N. D., 1970. Vertical distribution patterns of dissolved organic carbon in sea water and interstitial solutions. Oceanology, 10: 796-807.

Stein, R., 1986. Organic carbon and sedimentation rate- Further evidence for anoxic deep-water conditions in the Cenomanian/Turonian Atlantic Ocean. Mar. Geol., 72:199-209.

Stumm, W., and Morgan, J. J., 1981. Aquatic Chemistry. New York (Wiley).

Sugimura, Y., and Suzuki, Y., 1988. A high-temperature oxidation method for the determination of nonvolatile dissolved organic carbon in seawater by direct injection of a liquid sample. Mar. Chem., 24:105-131.

Suzuki, Y., Sugimura, Y., and Itoh, T., 1985. A catalytic oxidation method for the determination of total nitrogen dissolved in seawater. Mar. Chem., 16:83-97.

Toth, D. J., and Lerman, A., 1977. Organic matter reactivity and sedimentation rates in the ocean. Am. J. Sci., 277:265-285.

Wangersky, P. J., 1978. Production of dissolved organic matter. In Kinne, O. (Ed.), Marine Ecology, (Vol. 4): New York (Wiley).

Wangersky, P. J., and Zika, R. G., 1978. The analysis of organic compounds in sea water. N.R.C. Can., Mar. Anal. Chem. Stand. Prog. Tech. Rep. 3., 1-177.

Whelan, J. K., 1986. Geochemistry summary-Leg 96-The Mississippi Fan. In Bouma, A. H., Coleman, J. M., Meyer, A. W., et al., Init. Repts. DSDP, 96: Washington (U.S. Govt. Printing Office), 691698.

Whelan, J. K., and Tarafa, M., 1986. Organic matter in Leg 96 sediments: characterization by pyrolysis. In Bouma, A. H., Coleman, J. M., Meyer, A. W,. et al., Init. Repts. DSDP, 96: Washington (U.S. Govt. Printing Office), 757-766.

Whelan, J. K., Oremland, R., Tarafa, M., Smith, R., Howarth, R., and Lee, C., 1386. Evidence for sulfate-reducing and methane-producing microorganisms in sediments from Sites 618, 619, and 622. In Bouma, A. H., Coleman, J. M., Meyer, A. W., et al., Init. Repts. $D S D P$, 96: Washington (U.S. Govt. Printing Office), 767-775.

Date of initial receipt: 12 January 1988

Date of acceptance: 14 November 1988

Ms 113B-167 